\title{
An infant with Recurrent Infections -What should we think
}

\author{
R J Akhter ${ }^{1}$, B H N Yasmeen ${ }^{2}$, M L Nahar ${ }^{3}$
}

1 Dr. Rowshan Jahan Akhter Resident Medical Officer Dept. of Neonatology Dhaka Shishu (Children) Hospital

${ }^{2}$ Prof. Dr. B H Nazma Yasmeen Professor \& Head Dept. of Paediatrics Northern International Medical College

${ }^{3}$ Dr. Mst. Laizuman Nahar MPH Student SUNY, USA

\section{Correspondence} Dr. Rowshan Jahan Akhter Resident Medical Officer Dept. of Neonatology Dhaka Shishu (Children) Hospital e-mail : rowshanfairuz22@gmail.com
A 4 months old male child of a nonconsanguineous parents was admitted in Dhaka Shishu Hospital on 26.01.2016 with the complaints of cough and cold for 1 month, fever for 5 days, reluctant to feed for 2days. He had several histories of recurrent cough and fever for which he needed hospitalization. This was his $4^{\text {th }}$ hospital admission. He was on exclusive breastfed.

During admission we found that the child was febrile (temp- $102^{\circ} \mathrm{F}$ ), Tachypneic (R/R$68 / \mathrm{min}$ ). There was chest in drawing with bilateral coarse crepitation. Heart rate was148/minute without any added sound. Liver was just palpable. His weight was $4.8 \mathrm{~kg}$, height was $56 \mathrm{~cm}$ which was on $50^{\text {th }}$ centile and milestone of development was age appropriate. He was conscious and there were no features of meningial irritation.

With these features we diagnosed him as a Recurrent Bronchopneumonia patient.

Sequence of events according to time lineThe child was first admitted in Dhaka shishu Hospital on 23.11.15 at his 2 month of age with fever and convulsion and was diagnosed as pyogenic meningitis and treated with conventional antibiotics for 14 days. After improvement he was discharged on 05.12.16.

After 4 days of discharge he was again admitted to Hospital on 9.12.15 with the diagnosis of Pneumonia and treated with antibiotic for 7 days and then discharged.
On 28.1215 he was readmitted in hospital for the third time and diagnosed as Bronchopneumonia with UTI. This time child was evaluated for immunodeficiency and discharged on 11.01. 16.

Fifteen days after this episode he was admitted again on 26.01.16 and discharged on 02.02 .16 which described early.

\section{Investigations done in different admissions}

\begin{tabular}{|c|c|c|c|c|}
\hline \multicolumn{5}{|c|}{ CBC Report } \\
\hline Admission & $\stackrel{1^{\text {st }}}{\text { admission }}$ & $\begin{array}{c}2^{\text {nd }} \\
\text { admission }\end{array}$ & $\begin{array}{c}3^{\text {rd }} \\
\text { admission }\end{array}$ & $\begin{array}{c}4^{\text {th }} \\
\text { admission }\end{array}$ \\
\hline $\mathrm{Hb} \%$ & $8.5 \mathrm{gm} / \mathrm{dl}$ & $7.6 \mathrm{gm} / \mathrm{dl}$ & $12.8 \mathrm{gm} / \mathrm{dl}$ & $11.9 \mathrm{gm} / \mathrm{dl}$ \\
\hline WBC & $2900 / \mathrm{cmm}$ & $16300 / \mathrm{cmm}$ & $14000 / \mathrm{cmm}$ & $18200 / \mathrm{cmm}$ \\
\hline PLT & 270000 & 587000 & 300000 & 410000 \\
\hline \multicolumn{2}{|c|}{ Neutrophil $62 \%$} & $65 \%$ & $64 \%$ & $61 \%$ \\
\hline \multicolumn{2}{|c|}{ Lymphocyte33\% } & $34 \%$ & $28 \%$ & $36 \%$ \\
\hline
\end{tabular}

$\begin{array}{cccc}\text { Admission } & \begin{array}{c}\text { 2nd } \\ \text { admission }\end{array} & \begin{array}{c}\text { 3nd } \\ \text { admission }\end{array} & \begin{array}{c}\text { 4th } \\ \text { admission }\end{array} \\ \text { CXR } & \text { Right } & \text { Bi lateral } & \text { Right } \\ & \text { upper lobe } & \text { patchy } & \text { upper and middle } \\ & \text { consolidation } & \text { opacities } & \text { lobe consolidation }\end{array}$

CSF Analysis revealed features of pyogenic meningitis with high leukocyte count $\left(160 / \mathrm{mm}^{3}\right)$ and high protein count $(200 \mathrm{mg} / \mathrm{dl})$ on $1^{\text {stadmission }}$

Blood C/S revealed no growth in different occasions as patient got antibiotics before admission.

CRP was high in all admission.

Urine analysis showed growth of Enterococcus on 3rd admission

Echocardiography - Normal study

MT Test - Negative 


\section{Immunological Investigations}

Immunoglobulin assay

Immunoglobulin

\section{Result}

IgG

$4.85 \mathrm{gm} / \mathrm{L}$

Reference value

IgM

$\operatorname{Ig} \mathrm{A}$

$$
2.13 \mathrm{gn} / \mathrm{L}
$$

$0.23 \mathrm{gm} / \mathrm{l}$

$7-16 \mathrm{gm} / \mathrm{L}$

$0.4---2.3 \mathrm{gm} / \mathrm{L}$

$0.7-4.0 \mathrm{gm} / \mathrm{L}$

\begin{tabular}{|c|c|c|c|c|}
\hline \multicolumn{5}{|c|}{$\begin{array}{c}\text { Primary Immunodeficiency panel } \\
\text { Lymphocyte subset (TBNK) Analysis-Flow cytometry }\end{array}$} \\
\hline Markers & Result (\%) & $\begin{array}{l}\text { Ref. Value } \\
(3 m-12 m)\end{array}$ & $\begin{array}{l}\text { Result } \\
\text { cell/UL }\end{array}$ & $\begin{array}{l}\text { Ref. Value } \\
(3 m-12 m)\end{array}$ \\
\hline \multicolumn{5}{|l|}{ T cell Markers } \\
\hline $\mathrm{CD}^{+}$ & $63.91 \%$ & $51.8-74.2$ & 4474 & $2284-4776$ \\
\hline $\mathrm{CD}^{+}$ & $0.71 \%$ & $12.8-27.1$ & 50 & $524-1543$ \\
\hline $\mathrm{CD}^{+}, \mathrm{CD}^{+}+$ & $38.28 \%$ & $34.9-53.1$ & 2723 & $1523-3472$ \\
\hline $\mathrm{CD}^{+}{ }^{+}, \mathrm{CD} 8^{+}$ratio & & & 54.24 & $1.48-3.77$ \\
\hline \multicolumn{5}{|l|}{ B cell Markers } \\
\hline $\mathrm{CD} 19^{+}$ & $0.80 \%$ & $17-37.2$ & 57 & $776-2238$ \\
\hline \multicolumn{5}{|l|}{ NK cell Markers } \\
\hline $\mathrm{CD} 16^{+}, \mathrm{CD} 6^{+}$ & $13.59 \%$ & $4-15$ & 967 & $230-801$ \\
\hline \multicolumn{5}{|c|}{ Other cell Markers } \\
\hline CD45 & $50.39 \%$ & Up to $60 \%$ & 7112 & $3320-7006$ \\
\hline
\end{tabular}

\section{Treatment given}

During last three months several antibiotics were given like Injection Ceftazidim, Ceftriaxone, Flucloxacillin, Meropenem, syrup Clarithromycin.

Syrup cotrimoxazole was given as prophylactic antibiotic.

Besides antibiotics, Injection Pentaglobulin was given during his third hospital admission.

Our treatment plan-

- Prophylactic antibiotic for certain period.
- During infection antibiotic plus Pentaglobulin combindly can be given.

- Avoidance of live vaccine.

- Killed vaccines is safe, therefore these vaccination can be completed.

- We may give Stem cell transplantation (not available in our country)

- Gene Therapy (not available in our country)

- Patient should be kept under a routine Follow-up.

\section{Conclusion}

Serious infection or recurrent infections in early age need evaluation for primary immunodeficiency. Doctor's should think of this sorts of immunodeficiency cases.

\section{References}

1. Stephan JL, Vlekova V, Le Deist $F$ et al. Severe combined immunodeficiency: a retrospective single-center study of clinical presentation and outcome in 117 cases. J Pediatr 1993; 123:564-72.

2. Rosen FS. Severe combined immunodeficiency : A pediatric emergency. J Pediatr 1997; 130:324-25.

3. W.Pariyaprasert, P.Pacharn, N.Visitsunthorn et al., "Successful treatment of disseminated BCG infection in a SCID patient with granulocyte colony stimulating factor," Asian Pacific Journal of Allergy and Immunology, vol. 26, no. 1, pp. 71-75, 2008. View at Google Scholar View at Scopus

4. Grimbacher B, Schaffer AA, Holland SM, et al. Genetic linkage of hyper-IgE syndrome to chromosome 4. Am J Hum Genet 1999;65:735-44

5. Hsu AP, Davis J, Puck JM, et al. Autosomal dominant hyper IgE syndrome. GeneReviews, 